\title{
REVIEW
}

\section{You are what you eat, or are you? The challenges of translating high-fat-fed rodents to human obesity and diabetes}

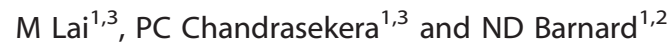

Obesity and type 2 diabetes mellitus (T2DM) are rapidly growing worldwide epidemics with major health consequences. Various human-based studies have confirmed that both genetic and environmental factors (particularly high-caloric diets and sedentary lifestyle) greatly contribute to human T2DM. Interactions between obesity, insulin resistance and $\beta$-cell dysfunction result in human T2DM, but the mechanisms regulating the interplay among these impairments remain unclear. Rodent models of high-fat diet (HFD)-induced obesity have been used widely to study human obesity and T2DM. With $>9000$ publications on PubMed over the past decade alone, many aspects of rodent T2DM have been elucidated; however, correlation to human obesity/diabetes remains poor. This review investigates the reasons for this translational discrepancy by critically evaluating rodent HFD models. Dietary modification in rodents appears to have limited translatable benefit for understanding and treating human obesity and diabetes due-at least in part-to divergent dietary compositions, species/strain and gender variability, inconsistent disease penetrance, severity and duration and lack of resemblance to human obesogenic pathophysiology. Therefore future research efforts dedicated to acquiring translationally relevant data-specifically human data, rather than findings based on rodent studies-would accelerate our understanding of disease mechanisms and development of therapeutics for human obesity/T2DM.

Nutrition \& Diabetes (2014) 4, e135; doi:10.1038/nutd.2014.30; published online 8 September 2014

\section{INTRODUCTION}

Type 2 diabetes mellitus (T2DM) is a rapidly growing global epidemic affecting $>371$ million people worldwide. ${ }^{1}$ In the United States, $>25$ million people have diabetes, and it is predicted that 1 in 3 Americans will be diabetic by year $2050 .^{2}$ Adult-onset T2DM is a complex multifactorial metabolic disorder influenced by genetic, lifestyle and environmental risk factors. Impaired glucose homeostasis in T2DM is primarily characterized by insulin resistance and $\beta$-cell dysfunction culminating in the morbidity (nephropathy, neuropathy, retinopathy and increased risk of cardiovascular disease) and mortality. One of the major risk factors for the development of insulin resistance and subsequent T2DM is obesity, another epidemic affecting 2.1 billion individuals worldwide. ${ }^{3}$ Obese individuals often have excess central visceral adiposity, a condition that contributes to a chronic increase in circulating free fatty acids and the resulting metabolites, such as diacylglycerol and ceramide. These metabolites in turn activate various signaling cascades that interfere with insulin signaling and $\beta$-cell function, further contributing to the gluco/lipotoxicity. ${ }^{4}$ Obesity also increases cardiovascular risk factors, such as dyslipidemia, hypertension and atherosclerosis. Therefore much of the research efforts over the past two decades have been dedicated to delineating the etiopathogenic mechanisms of obesity and diabetes.

A large number of animal models have been generated to study obesity and diabetes using species ranging from fruit flies to primates, including dogs, cats, pigs, rabbits, hamsters and squirrels to common rodent species, rats and mice. ${ }^{5,6}$ In general, T2DM is induced in experimental animals by surgical, chemical, dietary and genetic manipulations as well as combinations thereof. The most common obese models of T2DM are of spontaneous genetic origin (for example, naturally occurring mutations in leptin and leptin receptor) ${ }^{7}$ or experimentally induced by diet (for example, prolonged high-fat diet (HFD) feeding). ${ }^{7,8}$

A simple PubMed database search (using the keywords 'high fat diet' $^{\prime}$ and 'high fat diet and obesity' with the filters 'other animals' and 'publication dates') revealed that $>16000$ animal-based HFD studies have been published to date since the first article appeared on PubMed in 1964. The trend for animal-based HFD studies has increased tremendously since then-of the 16000 papers, $>9800$ papers were published in the past decade. Despite the wealth of information obtained from these animal-based HFD studies, the mechanisms regulating the interplay among obesity, insulin resistance and $\beta$-cell dysfunction in humans remain unclear. It appears that dietary modification in rodents has limited translatable benefit for understanding and treating human obesity and diabetes. The purpose of this review is to investigate the reasons for this translational discrepancy. This paper also addresses future directions necessary to conduct transnationally relevant human obesity and T2DM research.

\section{HFD: DIETARY OPTIONS}

The first description of HFD experiments dates back to the early 1940 s when rats fed an extreme HFD (70\% energy from fat) developed obesity with elevated basal and postprandial blood glucose levels. ${ }^{9,10}$ Another early diet was the 'cafeteria diet', a diet

\footnotetext{
${ }^{1}$ Physicians Committee for Responsible Medicine, Washington, DC, USA and ${ }^{2}$ Department of Medicine, George Washington University School of Medicine and Health Sciences, Washington, DC, USA. Correspondence: Dr PC Chandrasekera, Physicians Committee for Responsible Medicine, 5100 Wisconsin Avenue NW Suite 400, Washington, DC 20016, USA.

E-mail: cchandrasekera@pcrm.org

${ }^{3}$ These authors contributed equally to this work.

Received 24 February 2014; revised 18 June 2014; accepted 25 June 2014
} 
that included a variety of common, highly palatable human food that rodents were free to choose from (for example, cheese, ham, cookies, peanuts, chocolate and cakes). ${ }^{11}$ The utility of this cafeteria diet has diminished over the years as it is difficult to accurately quantify the nutrient intake with substantial variations in caloric content and types. ${ }^{11,12}$ Most researchers now use commercially available predefined HFDs encompassing a wide range of fat content and types. ${ }^{7}$ For example, Harlan Laboratories and Purina TestDiet provide a number of distinct HFD formulas, where the fat content ranges from $40 \%$ to $60 \%$. The fat types include saturated fatty acids, monosaturated fatty acids, polysaturated fatty acids and various combinations thereof, all typically derived from ingredients such as butter, pork fat, beef tallow, lard and various oils such as corn, coconut, cottonseed, soybean, olive, peanut, sesame, cocoa butter and fish oils. ${ }^{13,14}$

In addition to HFD, high-carbohydrate diets such as highfructose and high-sucrose diets are also used to induce features of the human metabolic syndrome in rodents. High-carbohydrate diets can be used alone or in combination with a HFD. For example, a high-sucrose (10-30\%) diet can be combined with high fat $(20-40 \%)$ to induce metabolic perturbations, such as increased body weight, abdominal fat deposition, hyperleptinemia, hyperinsulinemia, and hyperglycemia. ${ }^{15-17}$ High-fructose $(10-60 \%)$ is combined with high fat $(20-60 \%)$ to induce the symptoms such as increased body weight and the plasma concentrations of cholesterol, triglycerides, free fatty acids and leptin. ${ }^{16,18,19}$

HFD-fed rodents are often compared against control-diet-fed rodents in which a grain-based 'chow' diet made primarily from corn, wheat, oats and soybeans provides basic nutrition. ${ }^{20}$ Some researchers have made HFDs by simply adding fat to grain-based chow, but this chow-based HFD is not recommended as a high percentage of fat dilutes other ingredients in the diet, thereby making the diet deficient in micronutrients and macronutrients. ${ }^{7}$ Given the broad range of dietary modifications now utilized in obesity and T2DM research, it has not been possible to define the 'ideal' HFD nor generate a rodent model that can accurately mimic the human disease state. As discussed in the following sections, dietary modification in rodents appears to have limited translatability due-at least in part-to divergent dietary compositions, species/strain, sex and age variability, inter- and intra-laboratory variability in disease penetrance, severity and duration and lack of resemblance to the human obesogenic pathophysiology.

\section{HFD: MAJOR LIMITATIONS TO HUMAN TRANSLATABILITY}

HFD-feeding: effect of dietary components

A wide variety of methods are utilized in HFD studies, with no single method being comparable to the human experience. A multitude of diets with very different fatty acid compositions are included under the term 'high fat diet', and therefore the resulting phenotypes also vary considerably. Given the extensive literature on this topic, only select studies are presented below to illustrate the variable effects of dietary components and schemes on glucose regulation. For example, weight gain was reported to differ significantly between mice fed a HFD consisting of beef fat versus a HFD of canola oil (138\% more weight gain with beef fat compared with canola oil) despite $40.8 \%$ of energy from fat in both diets. ${ }^{21}$ In another study, weight gain in mice varied with the type of fat intake from oils containing different amounts of palmitic, linoleic and oleic acids: soybean $>$ palm $>$ or $=\operatorname{lard}>$ or $=$ rapeseed $>$ or $=$ safflower $>$ or $=$ perilla $>$ fish oil. ${ }^{22}$ More recent studies reiterate that even when protein and carbohydrate ratios are kept constant, weight gain in the two most commonly used mouse strains $(\mathrm{C} 57 \mathrm{BL} / 6 \mathrm{~J}$ and $\mathrm{A} / \mathrm{J})$ sharply contrasted between $60 \%$ HFD consisting of $100 \%$ saturated fatty acids and a $60 \%$ HFD consisting of $35 \%$ saturated, $43.4 \%$ monounsaturated and $15.9 \%$ polyunsaturated fatty acids. ${ }^{23}$ In addition to weight gain, the resulting metabolic manifestations can also differ with the type and amount of fat. For example, lard and olive oil have been shown to cause higher insulin resistance in rodents than coconut oil and fish oil. ${ }^{7,24}$ Moreover, despite similar energy intake, combination diets such as high-fat/high-sucrose diets can exert differential effects on insulin sensitivity and islet $\beta$-cell adaptation compared with HFD alone. ${ }^{25}$ Variations in fat-mediated metabolic perturbations and their impact on rodent studies are discussed in detail later.

Comparative dietary effects are generally made against chowbased control diets; however, variations in control diets can seriously impair data analysis. A study that examined HFD publications in the year 2007 revealed that only 14\% of the publications made the appropriate comparisons. ${ }^{26}$ The vast majority of these studies made conclusions regarding dietary effects by comparing grain-based chow with a predefined HFD, neglecting the differences in nutrient content in the two diets, which can cause unintended but significant metabolic disturbances. For example, the type of protein may modulate weight gain in rodents-rats fed soy diets have less hepatic lipid deposition, no hyperleptinemia and less overall weight than those fed casein diets. ${ }^{27}$ Other ingredients such as fructose can exacerbate weight gain and isoflavones (phytoestrogens) can influence fat deposition, plasma insulin, leptin, thyroid, estradiol and corticosterone levels, lipogenesis and lipolysis in rat adipocytes as well as consumptive behavior (food and water intake), learning and memory and anxiety-related behaviors in rodents. ${ }^{26}$ These results suggest that the effects of HFD are confounded by other components present in each diet, further hindering accurate data interpretation and extrapolation.

Even the standard 'control' animals may not be appropriate controls for obesity research as, in the words of the study author, these mice kept in controlled environments under a sedentary lifestyle with continuous access to food are 'metabolically morbid... obese, glucose intolerant, and on a trajectory to premature death'. ${ }^{28}$ Taken together, due to a broad range of dietary interventions confounded by other factors affecting overall metabolism and physiology, it has not been possible to define the 'ideal' HFD nor the ideal environment in which to conduct these experiments. Consequently, results reported among laboratories are not comparable even among the same species/strain, and reliable cross-species extrapolation is challenging.

\section{HFD-induced metabolic perturbations: sex, age and strain-} dependence

The commonly reported T2DM-related metabolic perturbations induced by HFD in rodents include weight gain and increased carcass lipid content, higher degree of insulin resistance and increased levels of plasma glucose, insulin, leptin, cholesterol and triglycerides. However, as discussed below, the development, severity and duration of these metabolic perturbations are highly variable due to several factors such as the age at which HFD feeding starts, duration of HFD feeding, sex and the rodent species as well as the strain used, ${ }^{29}$ rendering data analysis and extrapolation very difficult. Given the breadth of the literature on this topic, only representative examples are discussed below.

The age of onset and the duration of diet are important parameters when studying the metabolic syndrome in rodents. For example, in the most commonly used obesity-prone mouse strain C57BL/6J, 10-week-old mice displayed smaller increases in body weight, serum glucose, cholesterol and urea levels and higher increases in high-density lipoprotein levels than 54-weekold mice following the identical HFD for 12 weeks. ${ }^{30}$ When given a high-fructose or a HFD for 13 weeks, the HFD caused the largest changes in serum lipids and lipid accumulation in the liver and kidney in young (4-weeks old) rats, whereas the high-fructose diet 
increased visceral lipid stores, mean arterial pressure and heart rate in adult (12-weeks old) rats, and the metabolic perturbations became evident at different stages of the dietary intervention, suggesting that metabolic effects of diet vary considerably with age. ${ }^{31}$ Young mice (6-weeks old) were able to maintain normoglycemia in response to a HFD by increasing $\beta$-cell mass and $\beta$-cell proliferation, whereas older mice (7-8-month old) were not able to counter-regulate by the same mechanism and became diabetic. $^{32}$

The variability of metabolic perturbations among different rodent strains has been reported since the 1970s. Schemmel et al. $^{33}$ found that among seven different rat strains, HFD-induced weight gain ranged from $50 \%$ weight gain in Osborne-Mendel and Wistar-Lewis rats to $12 \%$ weight gain in S5B/P1 males to no weight gain in S5B/P1 females, indicating both strain- and sex-related differences. Similarly, the common mouse strains also range between obesity-prone and obesity-resistant. ${ }^{34} \mathrm{~A}$ more in-depth comparison between the two most common strains of mice (C57BL/6J and AKR/J) indicated that insulin sensitivity and regulation of glucose homeostasis differ markedly between these two strains: C57BL/6J mice display rapid weight gain (14.78 vs $3.74 \mathrm{~g}$ in control), hyperglycemia (132 vs $117 \mathrm{mg} \mathrm{dl}^{-1}$ in control), and insulin resistance (twofold less sensitive), whereas AKR/J have increased carcass lipid content (2916 vs $1006 \mathrm{mg}$ in control), severe impaired glucose tolerance and insulin resistance (5.5-fold less sensitive) but remained euglycemic ( $100 \mathrm{vs} 96 \mathrm{mg} \mathrm{dl}^{-1}$ in control). ${ }^{35}$ There were no factors that could account for this greater insulin resistance in AKR/J strain given that non-adipose tissue triglyceride accumulation was similar in both strains (143 in C57BL/6J vs $171 \mathrm{mg} \mathrm{dl}^{-1}$ in AKR/J, P>0.01). ${ }^{35}$ Of note, it has been shown that some strains have higher fasting plasma glucose levels and lower fasting plasma insulin levels regardless of dietary content. ${ }^{36}$ This is not so much an issue considering human T2DM patients also display a wide range of glucose intolerance and insulin resistance; however, the major limitation is that these data are not reproducible among research groups and therefore cannot be reliably extrapolated across species.

Levin et $a .^{37}$ reported that, in an outbred adult male SpragueDawley population, weight gain displayed a bimodal distribution pattern even though the rats were given the same HFD. Only half the rat population developed obesity, while the remainder showed no difference from control group. Similarly, female Wistar rats also showed a bimodal weight gain pattern on a HFD. ${ }^{38}$ As both Sprague-Dawley and Wistar rats are outbred strains, one might argue that the metabolic heterogeneity is due to the illdefined genetic nature of outbred colonies. ${ }^{39}$ However, similar metabolic variability is also found in C57BL/6J, the most commonly used inbred mouse strain used for HFD studies. Burcelin et $a^{40}{ }^{40}$ found that when C57BL/6J mice were on a HFD for 9 months, the development of obesity and diabetes was not uniform within the population-both lean and obese phenotypes developed on the HFD. More strikingly, it was found that mice that remained lean and non-diabetic after 9 months of HFD feeding actually had a significantly higher rate of glucose clearance than those mice in the control group. The wide scattered phenotypic differences cannot be explained by the variations in genetic background, because each C57BL/6J mouse has the identical genetic background. The variability in metabolic perturbations within a single population compromises any conclusions drawn from HFD studies, but no solution exists in the research community to overcome this critical issue.

Some researchers consider the variability among rodent strains to be similar to the ethnic diversity in the human T2DM population and argue that multiple phenotypic variations provide a selection pool for finding suitable models. ${ }^{41}$ However, in reality, this variability among strains causes more detriment than presumed benefit owing to irreproducible results. For example, even with the same C57BL/6J strain as a comparison baseline,
DBA/2 mice were observed as obesity-prone in one study ${ }^{36}$ but obesity-resistant in another. ${ }^{42}$ Similar discrepancies have been observed in $\mathrm{FVB} / \mathrm{N}$ mice as well. ${ }^{43,44}$ To date, only a few comprehensive studies using multiple strains have been published, and the vast majority of HFD studies are based on a single strain and single sex. It is unclear whether most researchers select their 'most suitable strain' based on thorough examination of the phenotypic variations reported. What is clear is that no single strain can represent a reliable model for human obesity and T2DM.

HFD-feeding: a comparative analysis of intra- and inter-laboratory variability

As discussed, HFD-induced metabolic syndrome in rodents is plagued by many confounding factors, including, but not limited to, the type of diet (and control diet), duration of exposure to diet, animal species and strain, age and gender of the animals, and the clinical manifestations developed and their definitions. Therefore it is inevitably challenging to find studies suitable for direct comparison. Nevertheless, it is important to determine whether HFD data from different laboratories (and within the same laboratory) can be reliably compared and extrapolated. In this regard, Table 1 illustrates comparisons made with six studies that used the same mouse strain, C57BL/6J.23,40,45-48

Among the six studies, the studies of Winzell and Ahren ${ }^{45}$ and of Reimer and Ahren ${ }^{47}$ have the most similar protocols: both studies used female wild-type C57BL/6J and started HFD at the age of 4 weeks with the same diet composition. However, even in studies from the same lab, presumably under the same protocol (that is, 58\% calories from fat), different observations have been made regarding the development of hyperinsulinemia (see 1 week versus 8 weeks in Table 1). It is difficult to explain this disparity, because these two experiments are from the same lab, presumably with the same protocols, with the only difference being the duration of study ( 1 year versus 8 weeks on HFD). Considering the duration of study, Sone and Kagawa ${ }^{46}$ conducted their study for 1 year, which is the same as Winzell and Ahren. ${ }^{45}$ However, C57BL/6J mice used in the two studies were of different sexes, age of onset of HFD feeding and diet compositions. The two studies reported inconsistent results regarding the progression of weight gain and hypersinsulinemia (Table 1), but it is difficult to explain the disparity because the two protocols differ in many aspects except for the duration of study. Considering that dietary effects vary greatly within species and between closely related rodent species such as mice and rats, it is challenging to extrapolate to a distantly related species like Homo sapiens.

In addition to the biological variability, technical variability inherent in the assays and techniques used for measuring various parameters of the metabolic syndrome in rodents further complicates data acquisition, analysis and interpretation. Assays such as glucose and insulin tolerance tests and hyperglycemic and hyperinsulinemic-euglycemic clamps have inherent technical difficulties confounded by procedural factors affecting glucose metabolism, such as anesthesia, site and volume of blood sampling, fasting duration and even ambient lighting and time of day. ${ }^{49}$ For example, anesthesia can induce hyperglycemia in mice and influence the assessment of glucose metabolism. ${ }^{50}$ Tail-bleed blood sampling of $100 \mu \mathrm{l}$ can cause stress and lead to increased catecholamine and basal glucose levels compared with same volume of artery blood sampling. ${ }^{49}$ In addition, rodent genetic background and environmental interactions (for example, housing facilities) can affect clinical chemical and hematological parameters, such as glucose, cholesterol, triglycerides, creatinine, uric acid, hemoglobin, red and white blood cell counts and platelet counts. ${ }^{51}$ Fasting duration, especially an 18-h overnight fast, can result in the loss of total body, lean and fat masses as well as hepatic glycogen levels while a 5 -h fast can result in increased 
Table 1. Inter- and intra-laboratory variability among six HFD studies using C57BL/6J mouse models

\begin{tabular}{|c|c|c|c|c|c|c|}
\hline HFD in C57BL/6J & $\begin{array}{l}\text { (a) Winzell and } \\
\text { Ahren }^{45}\end{array}$ & $\begin{array}{l}\text { (a) Reimer and } \\
\text { Ahren }^{47}\end{array}$ & (b) Sone and Kagawa ${ }^{46}$ & (c) Wu et al. ${ }^{48}$ & $\begin{array}{l}\text { (d) Gallou-Kabani } \\
\text { et al. }\end{array}$ & (e) Burcelin et al. ${ }^{40}$ \\
\hline Sex & Female & Female & Male & Male & Both sexes & Male \\
\hline Control diet & $11.4 \%$ fat & $11.4 \%$ fat & $4 \%$ fat & $4.3 \%$ fat & $10 \%$ fat & $12 \%$ fat \\
\hline HFD: diet & $58 \%$ fat & $58 \%$ fat & $40 \%$ fat & $35.2 \%$ fat & $60 \%$ fat & $72 \%$ fat \\
\hline HFD: oil & Lard & Not specified & Beef tallow & Lard & Various $^{\mathrm{a}}$ & Corn oil and lard \\
\hline Hyperglycemia & $\begin{array}{l}\text { Hyperglycemia } \\
\text { evolved after } \\
1 \text { week on HFD }\end{array}$ & $\begin{array}{l}\text { Hyperglycemia } \\
\text { evolved after } \\
1 \text { week on HFD }\end{array}$ & Hyperglycemia & $\begin{array}{l}\text { Mild hyperglycemia. } \\
\text { Lost at week } 40\end{array}$ & Hyperglycemia & $\begin{array}{l}\text { Heterogeneous } \\
\text { responses }\end{array}$ \\
\hline Hyperinsulinemia & $\begin{array}{l}\text { Hyperinsulinemia } \\
\text { developed after } \\
1 \text { week on HFD }\end{array}$ & $\begin{array}{l}\text { Hyperinsulinemia } \\
\text { developed after } \\
8 \text { weeks on HFD }\end{array}$ & $\begin{array}{l}\text { Hyperinsulinemia at } \\
\text { month } 4 \text { but no difference } \\
\text { from control at month } 12\end{array}$ & Hyperinsulinemia & $\begin{array}{l}\text { Hyperinsulinemia } \\
\text { only found in } \\
\text { males }\end{array}$ & $\begin{array}{l}\text { Heterogeneous } \\
\text { responses }\end{array}$ \\
\hline
\end{tabular}

insulin sensitivity. ${ }^{52}$ In humans, prolonged fasting impairs insulinstimulated glucose utilization, but the opposite is true for rodents. ${ }^{53}$ Metabolic studies in rodents have shown that changes to circadian rhythm can also affect glucose metabolism. ${ }^{54}$ It is clear that various factors associated with the technical procedures for assessing glucose metabolism in rodents significantly contribute to inconsistent results, rendering inter-species extrapolation an arduous task.

HFD-feeding: in combination with chemical, surgical and genetic manipulations

HFD is often used in combination with chemical, surgical and genetic modes of T2DM induction, but limitations inherent in these methods have severely restricted the ability to interpret data in conjunction with HFD feeding. For chemical induction, the cytotoxic glucose analogs alloxan and streptozotocin are commonly used to induce irreversible pancreatic $\beta$-cell destruction. ${ }^{55}$ High-dose streptozotocin destroys most of the endogenous $\beta$ cells, thereby decreasing insulin secretion substantially (a condition similar to T1DM), whereas low-dose streptozotocin induces mild impairment of insulin secretion (a condition similar to T2DM). ${ }^{56,57}$ Chemical induction of a T2DM-like state has its own disadvantages and combining that with HFD feeding creates further complications hindering reliable data interpretation and extrapolation. For example, the dosage and the number of streptozotocin injections considerably vary among studies: in rats, dosage can range from $90 \mathrm{mg} \mathrm{kg}^{-1}$ (Eiki et al. ${ }^{58}$ ) to $30 \mathrm{mg} \mathrm{kg}^{-1}$ (Cao et al. $^{59}$ ) for single injections while the number of injections can range from two (Ding et al..$^{60}$ ) to four (Li et al..$^{61}$ ) with the dosage ranging from $5 \mathrm{mg} \mathrm{kg}^{-1}$ (Li et al. ${ }^{61}$ ) to $30 \mathrm{mg} \mathrm{kg}^{-1}$ (Zhang et $a l^{62}$ ). This in turn leads to highly variable fasting blood glucose levels (from 5 to $25 \mathrm{mmoll}^{-1}$ ), indicating different degrees of pancreatic $\beta$-cell destruction. ${ }^{62}$ Such discrepancies make it difficult to compare data even among rodent studies.
In addition, streptozotocin and alloxan can cause extrapancreatic genotoxic and cytotoxic effects, including the disruption of the hypothalamic-pituitary-gonadal axis ${ }^{63,64}$ and changes in hyperglycemia-unrelated hepatic gene expression, ${ }^{65}$ making it difficult to distinguish the effect caused by pancreatic cytotoxicity from those caused by extrapancreatic sites. The addition of age, sex and strain-dependent HFD feeding atop already complicated chemical T2DM induction renders intra- and inter-species extrapolation impossible.

The most common mode of surgical induction of T2DM is pancreatectomy, and ventromedial hypothalamus (VMH) lesions are sometimes used as well. The standard partial (up to 90\%) or complete pancreatectomy often produces non-obese diabetic models where the extent of pancreatectomy governs the severity of diabetes. ${ }^{5}$ Data interpretation and extrapolation from pancreatectomy models are limited by many confounding factors. First of all, human T2DM is not caused by a sudden loss of the pancreas; rather it is actually due to progressive loss of $\beta$-cell function in combination with insulin resistance. Second, in rodents, pancreatectomy often evokes robust cellular responses that lead to $\beta$-cell regeneration giving full recovery of $\beta$-cell mass even after $40-60 \%$ partial pancreatectomy ${ }^{66,67}$ and up to $200 \%$ increase in $\beta$-cell number in the pancreatic remnants following $90 \%$ pancreatectomy in rats. ${ }^{68}$ Unlike in rodents, partial pancreatectomy does not result in $\beta$-cell regeneration in humans, ${ }^{69}$ due to differences between human and rodent $\beta$-cell replication mechanisms. ${ }^{70}$ Although pancreatectomy is a common surgery used to induce T2DM in rodents, combining it with HFD only adds to the complexity-removal of the exocrine pancreas substantially reduces the production of most enzymes required for intestinal lipid absorption and lipolysis, making it difficult to obtain reliable results in HFD-fed pancreatectomized animals. ${ }^{71}$ These models also lack counter-regulatory mechanisms imposed by glucagon, thereby adding to the pathophysiological complexity. 
VMH lesions are generally induced by administration of monosodium glutamate or direct electrical shock to create bilateral destruction of the ventromedial and arcuate hypothalamic nuclei. This leads to obesity primarily by hyperphagia and lack of control between energy absorption and expenditure governed by leptin, neuropeptide $\mathrm{Y}$ and insulin feedback mechanisms. ${ }^{72}$ Phenotypic variability in $\mathrm{VMH}$ rodent models is exacerbated by HFD feeding. For example, VMH rats fed the same HFD develop hyperglycemia at different rates. ${ }^{73}$ Given the unnatural induction of hyperglycemic conditions by $\mathrm{VMH}$ lesions and the variable metabolic perturbations present even within a single rodent strain on a HFD, ${ }^{37}$ it is difficult to extrapolate these data to human obesity and diabetes.

HFD is often combined with genetically modified models (transgenic, knockout, knock-in and overexpression models), where an overt diabetic phenotype may not appear until obesity is induced by HFD. ${ }^{74}$ Regardless of the reason for dietary modification, genetically modified mice also display age-, sexand strain-dependent variability in disease phenotype with or without HFD induction (Table 2). A prime example of this comes from knockout mouse models of an islet-enriched zinc transporter, ZnT8, thought to reside in insulin secretory granules to modulate proper insulin maturation, storage and secretion. Three independent studies published in 2009 alone provided evidence that mice lacking the gene encoding ZnT8 develop an increased risk for diabetes; however, there were intriguing differences among these animal models. As shown on Table 2, these studies reported age-, sex-, strain- and diet-dependent phenotypic variability (for example, glucose tolerance, insulin secretion, body weight, etc.), ${ }^{75-77}$ even when two colonies of the same ZnT8 null were maintained in two separate laboratories with the primary difference being the number of times the knockouts were backcrossed into C57BL/6J background (three times in the Toronto, Canada colony and two times in the London, UK colony). ${ }^{75}$ Because of the highly variable results observed from the mixed genetic backgrounds in these studies, another group later generated ZnT8 knockouts on a pure genetic background (C57BL6/J) and concluded that ZnT8 does not have a substantial impact on mouse physiology. ${ }^{78}$ In addition, even the in vitro insulin secretion from ZnT8 knockout mouse islets varied from no effect $^{76}$ to decreased effect ${ }^{77}$ to enhanced insulin secretion. ${ }^{75}$ Furthermore, global knockouts of ZnT8 were shown to be more susceptible to diet-induced obesity compared with tissue-specific knockout mice. ${ }^{79}$ Following all these efforts to generate and characterize ZnT8 mouse models, a recent study involving 150000 people carrying ZnT8 mutations revealed that mouse results are not congruent with the phenotype observed in humans: in marked contrast to the animal models where gene ablation resulted in increased risk for T2DM, protein-truncating loss-offunction mutations actually protect humans from developing T2DM. $^{80}$

There are many other examples of highly disparate results arising from the combination of HFD feeding with genetic modification. For example, loss of GPR40 (a G-protein-coupled receptor expressed predominantly in pancreatic islets mediating free fatty acid-induced insulin secretion) protects mice from HFDinduced diabetes, ${ }^{81}$ whereas the same gene ablation fails to protect mice from HFD-induced diabetes in another study. ${ }^{82}$ Although disparity in GPR40 studies arose on a C57BL/6 J background, metabolic phenotypes appear to differ between strains of transgenic/knockout mice with identical genetic mutations (for example, insulin receptor and insulin receptor substrate-1 knockout mice). ${ }^{83,84}$ When HFD is combined with genetic modification in both sexes, the disparate phenotypes seen in males and females have forced studies to draw conclusions mainly from one sex ${ }^{85-88}$ - many HFD studies only include one sex in their experiments to avoid sex-related differences. There have even been instances where effects of a HFD diminished with time, as has been shown for low-density lipoprotein receptor knockout mice on a C57BL/6J background who lost their lard-based HFDinduced mild hyperglycemia after 40 weeks. ${ }^{48}$ This is rather unexpected as C57BL/6J is reported to develop more severe metabolic disturbances over other strains, such as AKR/J and $A / J$ mice. ${ }^{35}$ These studies beg the question, if a model using one rodent age, sex, strain and diet type cannot be extrapolated to another cohort of the same species, how much confidence can there be in extrapolating across the species barrier to humans?

In addition to the effect of dietary modification, primary and secondary effects of the genetic manipulation itself may heavily influence the observed phenotype. For example, genetic manipulation does not result in an observable phenotype due to the presence of compensatory mechanisms (for example, SUR1 knockout mice), or presents a more exaggerated phenotype than what may be present in the polygenic T2DM state (for example, glucose transporter 2 null mice). ${ }^{70}$ Gene inactivation is effective throughout development, and therefore it is difficult to distinguish phenotypes arising from developmental defects from those directly resulting from impaired glucose homeostasis. Without the ability to confidently differentiate between knockout effect, compensatory effect and developmental effects, genetically manipulated models offer limited value when combined with HFD feeding, where all of these issues are further exacerbated by HFD-dependent sex, age and strain variability. Taken together, HFD feeding with chemical, surgical and genetic manipulation further limits the ability of rodent models to accurately mimic human obesity and diabetes.

HFD-induced obesity and diabetes in rodents: relevance to human pathophysiology

In order for a rodent model to have relevance to human disease, it should effectively recapitulate the natural history, pathophysiology and complications in a manner similar to what is observed in humans. However, this does not appear to be the case for HFD-fed rodents trying to mimic human obesity and diabetes. First of all, the large number of diets used for HFD studies in rodents may not appropriately represent the general human diet, particularly the 'obesogenic' Western diet. For example, the US population on average consumes $30-40 \%$ dietary fat regardless of weight (whether normal, overweight or obese)..$^{89,90}$ Therefore, using $40-60 \%$ fat (that is, values significantly $>40 \%$ ) in rodent dietary interventions is too extreme compared with the average Western diet. Moreover, human diets are much more complex than the carefully formulated rodent HFDs. According to the US National Cancer Institute, human foods and beverages can be divided into 97 categories, such as grain-based desserts, pasta and pasta dishes, beef and beef-mixed dishes and so on, with many subcategories. ${ }^{91}$ Humans also consume alcohol and excessive sodium, which are not found in rodent feed, but affect human pathophysiology. Therefore rodent diets differ from the diet that lead to human obesity and diabetes and associated complications. $^{16}$

Apart from the actual diet, consumptive behavior also differs between rodents and humans. Humans tend to have stress, emotions and cultural factors that affect their access to food, selection of diet and feeding behavior. ${ }^{92}$ For example, uncontrolled food intake can lead to overeating due to complex psychological factors in humans, ${ }^{93}$ which cannot be accurately mimicked in rodent models. In some rodent models, disruption of leptin signaling is the primary cause of hyperphagia rather than feeding behavior governed by emotional factors. Furthermore, unlike humans, mice consume most of their food at night. ${ }^{94}$ As a result, the routine overnight fasting of $16-18 \mathrm{~h}$ is an unhealthy long period of time that provokes a catabolic state capable of nearly depleting liver glycogen stores, a state more akin to starvation in mice. ${ }^{95}$ In lean mice, overnight fasting can reduce 
Table 2. Variability among studies in which genetic modification is combined with HFD

ZnT8-/-: Colony derived in France on a mixed Age- and sex-dependence: in the Toronto colony, elevated FPG and Gl in male mice at 6 weeks of $129 \mathrm{SeVe} / \mathrm{C} 57 \mathrm{BL} / 6 \mathrm{~J}$ background and maintained age, but not at 12 weeks; normal FPG, but Gl at both ages in females; in the London colony, male in Toronto and London ${ }^{75}$ mice had Gl at both ages, but female mice had normal glucose tolerance at 12 weeks Strain-dependence: mixed 129SvEv/C57BL/6J background; Toronto colony was backcrossed $3 \times$ and the London colony was backcrossed $2 \times$ on to C57BL/6J (possible explanation for age- and sex-dependence)

Diet-dependence: HFD increased body weight in ZnT8-/ - compared with wild type; increased fasting plasma insulin levels In vitro: increased basal insulin release in ZnT8-/- islets

ZnT8-/-: Colony derived in France on a mixed Age-dependence: FPG and insulin levels unchanged at ages 6, 12, 25 weeks and 1 year. No $129 \mathrm{SeVe} / \mathrm{C} 57 \mathrm{BL} / 6 \mathrm{~J}$ background and maintained in Leuven ${ }^{76}$

difference in insulin sensitivity at 12 weeks

Sex-dependence: No apparent sex differences; only slight (but statistically significant) change in glucose tolerance at 6 weeks in female $\mathrm{ZnT} 8-/-$

Strain-dependence: mixed 129SvEv/C57BL/6J background; overall metabolic abnormalities mild compared with Nicolson et al. ${ }^{75}$

Diet-dependence: normal glucose tolerance on standard diet; HFD increased body weight by $10 \%$; mild Gl at 10 weeks on HFD; overt diabetes (blood glucose $>14 \mathrm{~mm}$ ) in $50 \%$ of ZnT8-/In vitro: no change in glucose-induced insulin secretion in ZnT8 knockout islets

ZnT8-/-: Mixed 129SeVe/ C57BL/6J background $^{77}$ Age-dependence: no change in body weight; blood glucose unchanged at 16 weeks of age Sex-dependence: compared with females, metabolic parameters such as plasma insulin, glucose, triglycerides and cholesterol levels were higher in males, but glucagon levels were lower in males Strain-dependence: mixed 129SeVeBrd/ C57BL/6J background; FPG unaltered; decreased insulin levels; no impairment in glucose clearance; overall mild metabolic phenotype

Diet-dependence: experiments conducted only on standard control diet In vitro: glucose-induced insulin secretion markedly decreased in ZnT8 knockout islets

ZnT8-/-: Pure C57BL/6J background ${ }^{78}$ Age-dependence: normal glucose tolerance at $\sim 20-22$ weeks, but a small impairment in glucose tolerance in younger ( $\sim 4$ weeks old) male mice; HFD studied only at $40-50$ weeks of age Sex-dependence: normal fasting insulin levels in males; reduced fasting plasma insulin levels, but no change in FPG levels in females

Strain-dependence: pure C57BL/6J background; no change in glucose tolerance; female phenotype appears to be less dependent on 129SvEv-specific modifier genes while male phenotype appears to be heavily influenced by $129 \mathrm{SvEv}$-specific modifier genes Diet-dependence: decreased plasma insulin levels in males; no diabetic phenotype-40-50week-old mice protected from HFD-induced obesity

In vitro: no change in glucose-induced insulin secretion

ZnT8-/-: Mixed 129SeVe/ C57BL/6J Global and $\beta$-cell-specific deletions of ZnT8-global knockouts more susceptible to HFD-induced obesity compared with tissue-specific knockouts. Global knockouts became obese, hyperglycemic, hyperinsulinemic, insulin resistant and glucose intolerant compared with littermate controls; in contrast, $\beta$-cell-knockouts had impaired glucose tolerance, though similar body weights, compared with littermate controls

Abbreviations: FPG, fasting plasma glucose; Gl, glucose intolerance; HFD, high-fat diet; ZnT8 - / - , homozygous knockout of zinc transporter type 8 encoded by S/C30A8 gene. This table summarizes a representative example of the variability commonly found in studies in which a genetic manipulation is combined with HFD feeding. Although there are many other studies that would fit these criteria, due to length limitations, only select examples are described in this review.

lean body mass by $\sim 15 \%{ }^{52}$ In addition, prolonged fasting in mice enhances insulin-stimulated glucose utilization, whereas it is impaired in humans. ${ }^{49}$ Moreover, mice can undergo reduced metabolic rate (physiological state of torpor), whereas humans cannot. $^{94}$

These metabolic stresses are further exacerbated by the ambient temperature in general laboratory housing $\left(18-22^{\circ} \mathrm{C}\right.$, which is well below their critical thermoneutrality point of $\sim 30^{\circ} \mathrm{C}$. This chronic thermal stress causes mice to increase their metabolism by increasing food intake by as much as 50\%, ${ }^{96-99}$ further skewing data. Controlled light/dark cycles adapted for human convenience (that is, typical 0800-1700 work hours) could have negative influences on mouse physiology, as changes to circadian rhythms can affect glucose metabolism. ${ }^{49}$ In general, rodents have higher metabolic rates governed by much higher heart rates ranging from 350 to 550 beats $\mathrm{min}^{-1}$, whereas humans have lower metabolic rates accompanied by lower heart rates around 70 beats $\min ^{-1}$.

Translatable rodent models of T2DM should accurately mirror the etiopathology of the human condition; however, this is rarely the case with the vast majority of rodent models, including HFD models. In humans, a complex genetic background interacts with environmental factors leading to progressive disease development, which occurs over a long period of time on the order of years to decades. ${ }^{100}$ In contrast, most rodent HFD studies last only a few weeks (and a few months at most) as rodents can develop obesity and insulin resistance after 2 weeks on a HFD, albeit to varying degrees. ${ }^{7}$ Because of rapid unnatural disease induction, HFD models often lack (or incorrectly mimic) T2DM-associated vascular complications, which develop over many years, usually starting long before the clinical diagnosis of overt hyperglycemia in humans. Macrovascular complications (primarily described as cardiovascular complications) are the leading cause of morbidity and mortality, which occur predominantly via accelerated atherosclerosis in humans. By contrast, rodents show resistance to high low-density lipoprotein cholesterol, atherosclerosis, platelet aggregation and hypertension. ${ }^{70}$ In a study examining the association between atherosclerosis and T2DM in a HFD rodent model, no difference exists between the control and the HFD group in all indicators of atherosclerosis despite the presence of metabolic perturbations such as hyperinsulinemia, hyperglycemia and obesity. $^{48}$ 
The main microvascular complications that occur in humans are nephropathy, neuropathy and retinopathy; however, reliable rodent models that can effectively recapitulate these human complications are still lacking, especially with HFD-induced models. For example, advanced proliferative diabetic retinopathy characterized by intravetreal neovascularization-the leading clinical feature causing blindness in humans worldwide-has not been successfully displayed in a single animal model of T2DM, much less HFD-fed rodents. ${ }^{101}$ For diabetic nephropathy, only early signs of renal damage are usually observed even in the longterm diet-induced models of T2DM. ${ }^{102}$ In terms of diabetic neuropathy, rodents may develop some peripheral nerve functional abnormalities but do not develop structural abnormalities seen with human diabetic neuropathy. ${ }^{70,103,104}$ Taken together, HFD rodent models display variable and partial features, often resembling only the early stages of human vascular complications. Therefore, these models have limited translatable benefit, and overestimation of these data can lead to inaccurate delineation of disease mechanisms in humans. Furthermore, drugs tested to be effective in models displaying only early signs of vascular complications may not work as effectively for the general human population that routinely manifests advanced disease phenotypes.

It appears that even functional genomics may not be reliably translated from HFD models to the human state. In a functional genomics study, comparing differential gene expressions in HFD rats and obese humans indicated that there is only minimal overlap of the differentially expressed genes between the HFD versus control rats and obese versus non-obese human comparisons. The study found that genes in both fatty acid metabolism and oxidation showed different regulation patterns between obese human and rats. ${ }^{105}$ The discrepancies in differential gene expression between HFD rats and obese humans, especially in the metabolic pathways, further decrease the applicability of HFD rodent models to human obesity and T2DM research. In addition, species differences at every level of glucose regulation (from gene expression to the maintenance of whole-body glucose homeostasis) further restrict the ability to extrapolate data from rodent HFD models to human obesity and diabetes. ${ }^{70}$

\section{SUMMARY AND FUTURE PERSPECTIVES}

A combination of several techniques has enabled the creation of a large number of animal models with varying obesogenic/ diabetogenic phonotypes. However, it is clear that the natural history and metabolic characteristics of the human condition cannot be effectively recapitulated in a single model or even a combination of these animal models. Induction of T2DM-like features with HFD feeding is very common in research, but major limitations significantly reduce the translatability of rodent-based dietary intervention data to human disease mechanisms and treatment options. Despite the current literature describing many thousands of diet-induced rodent studies, the question of which HFD is best to model the metabolic syndrome seen with human obesity still remains unanswered. Based on this body of literature that provides ample evidence showing even the same rodent (for example, Sprague-Dawley rat) from three different vendors (for example, Harlan, Charles River and Simonsen) can display substantial phenotypic differences with respect to metabolism, ${ }^{106}$ it is not possible to define a recipe for the 'ideal' HFD, dietary components and control diet capable of yielding the best and most reproducible results in rodents, nor the characterization of metabolic perturbations based on an exact dietary composition, duration of diet and age of onset of diet due to many confounding factors such as species, strain, age, sex, housing environment and husbandry practices and biochemical/physiological assessment techniques (Figure 1). Moreover, animal studies are biased with respect to experimental design and data reporting, with excess significance and overoptimistic translational
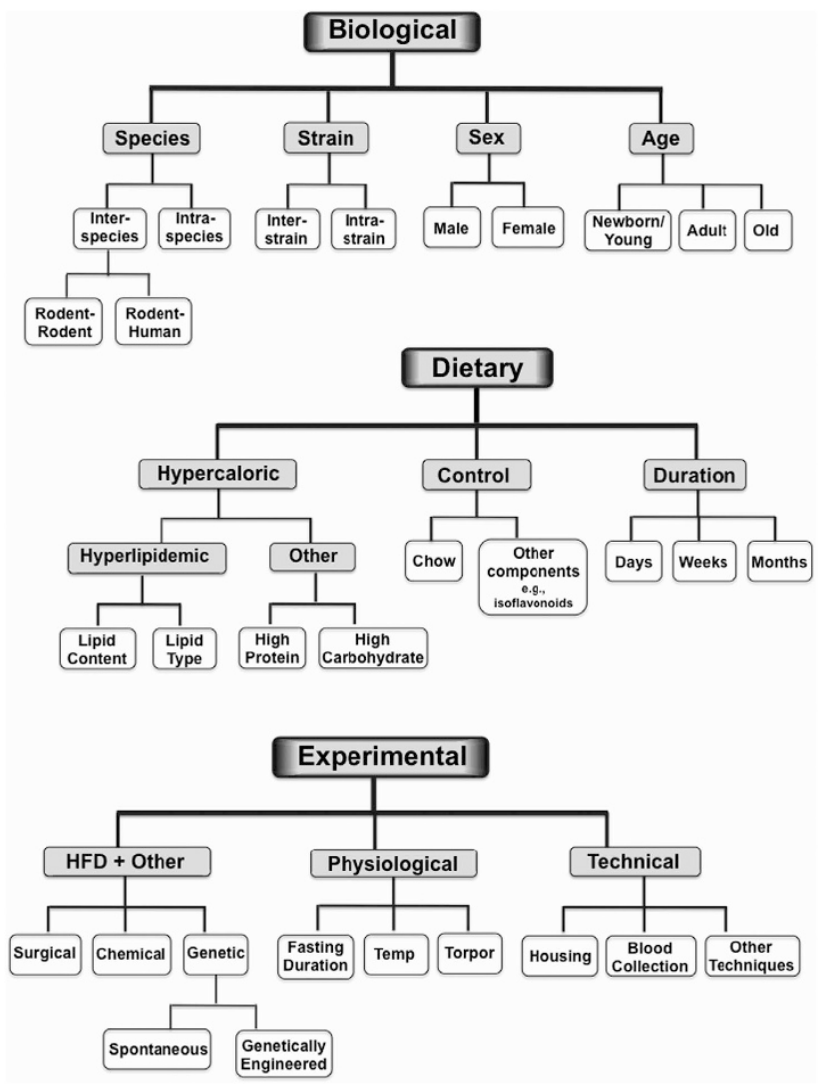

Figure 1. Primary factors contributing to the variability observed in HFD-fed rodent models. Variability observed within and among laboratories using HFD-based rodent models can be broadly classified into biological, dietary and experimental variability primarily arising from factors such as species, strain, sex, age, HFD fat content and type, other dietary components, control diet and duration of HFD feeding. This variability is further exacerbated when combined with chemical, surgical and genetic manipulations as well as physiological and environmental factors affecting data acquisition.

efficacy widely reported in the literature. ${ }^{107,108}$ Specious extrapolations and overstated significance of rodent studies have hindered the outcome of human therapeutics. Consequently, only a few anti-diabetic drugs are currently available on the market despite the substantial amount of information acquired thus far from animal studies of obesity and diabetes. ${ }^{70}$ It seems clear that research efforts must be redirected to studying human T2DM using human-based methods in order to acquire human-relevant information.

The limitations of animal models suggest the value of studying human glucose biology directly. Obesity and T2DM can be studied from gene expression to whole-animal physiology to environmental level using human-based methods. ${ }^{70}$ Utilization of a broad range of human-based methods would enable the researcher to reliably capture the whole spectrum of the polygenic, multifactorial human metabolic syndrome and T2DM. Acute and longterm effects of diet composition can be directly studied in humans on a specific dietary regime. For example, the identity of the molecular defect(s) underlying obesity-induced insulin resistance can be studied from skeletal muscle biopsy samples, and wholebody insulin sensitivity can be studied via hyperinsulinemiceuglycemic clamp from human subjects on a given dietary regime. ${ }^{109,110}$ Proteomic analysis of human skeletal muscle ${ }^{111}$ presents a dynamic pattern of protein abundance in insulin 
resistance, serving as a basis of novel hypothesis testing for molecular mechanistic studies.

Understanding disease etiopathogenesis is crucial for the development of new therapeutic interventions, but human environmental influence on disease ontogeny cannot be recreated in a laboratory setting nor can they exert the same effect on another species. Therefore studies in epigenome-wide DNA methylation profiling of pancreatic islets can help link epigenetics to T2DM and identify differential methylation in several T2DMassociated genes. ${ }^{112}$ A number of human epidemiological studies have resulted in the identification of dietary risk factors in various human populations, such as Pima Indians, Chinese and other ethnic groups. ${ }^{113-117}$ Recent studies have shown that diet and obesity may even have a microbial component. For example, intestinal microbiome gene profile has been shown to differ between lean and obese individuals on different diets, ${ }^{118}$ and the human gut microbial profile has been linked to human insulin action. ${ }^{119}$ Given that diet can modify factors like gut microbiota and subsequent influence on host metabolism, it is crucial to obtain data directly relevant to the human species, as such factors can differ significantly between rodents and humans. Further refinement of existing methods and the development of novel human-based research methods will transform obesity and T2DM research in a human-relevant manner.

Dietary modification in rodent models has limited translatable benefit for understanding the pathogenesis of human obesity and diabetes. The information obtained from the HFD studies are often confined to the species and strain, and even to simply one sex, rather than being applicable to the human disease state. It is, therefore, necessary to dedicate future research efforts to obtaining transnationally relevant data, specifically human data, rather than findings based on rodent studies. Redirecting biomedical research back to humans is clearly the way to efficiently deal with the current obesity and diabetes epidemics.

\section{CONFLICT OF INTEREST}

The authors declare no conflict of interest.

\section{REFERENCES}

1 International Diabetes Federation. IDF Diabetes Atlas, 5th edn. International Diabetes Federation: Brussels, Belgium, 2012. Available from http://www.idf.org/ diabetesatlas.

2 Centers for Disease Control and Prevention. National Diabetes Statistics Report: Estimates of Diabetes and Its Burden in the United States, 2014 US Department of Health and Human Services, Centers for Disease Control and Prevention: Atlanta, GA, USA, 2014.

3 U.S. Department of Agriculture and U.S. Department of Health and Human Services. Dietary Guidelines for Americans, 2010, 7th edn. Government Printing Office: Washington, DC, USA, 2010.

4 Kahn SE, Hull RL, Utzschneider KM. Mechanisms linking obesity to insulin resistance and type 2 diabetes. Nature 2006; 444: 840-846.

5 Islam MS, Loots du T. Experimental rodent models of type 2 diabetes: a review. Methods Find Exp Clin Pharmacol 2009; 31: 249-261.

6 Srinivasan K, Ramarao P. Animal models in type 2 diabetes research: an overview. Indian J Med Res 2007; 125: 451-472.

7 Buettner R, Schölmerich J, Bollheimer LC. High-fat diets: Modeling the metabolic disorders of human obesity in rodents. Obesity 2007; 15 798-808.

8 Wang B, Chandrasekera PC, Pippin JJ. Leptin- and leptin receptor-deficient rodent models: relevance for human type 2 diabetes. Curr Diabetes Rev 2014; 10: $131-145$.

9 Samuels LT, Reinecke RM, Ball HA. Effect of diet on glucose tolerance and liver and muscle glycogen of hypophysectomized and normal rats. Endocrinology 1942; 31: 42-45.

10 Samuels LT, Gilmore RC, Reinecke RM. The effect of previous diet on the ability of animals to do work during subsequent fasting: one figure. J Nutr 1948; 36 639-651.

11 Sclafani A, Springer D. Dietary obesity in adult rats: similarities to hypothalamic and human obesity syndromes. Physiol Behav 1976; 17: 461-471.
12 Tschop M, Heiman ML. Rodent obesity models: an overview. Exp Clin Endocrinol Diabetes 2001; 109: 307-319.

13 Harlan Laboratories. Teklad Custom Research Diets - Diet Induced Obesity 2008 [cited 2013]. Available from http://www.harlan.com/download.axd/ 3a69f6f83e174fff84b859f746b1b0fd.pdf.

14 Purina TestDiet I. Diet Induced Obesity Diets Richmond. Purina TestDiet, Inc: Indiana, USA, 2013. [cited 2013]. Available from http://www.testdiet.com/Diets/ High-Fat-DIO/index.html.

15 Murase T, Mizuno T, Omachi T, Onizawa K, Komine $\mathrm{Y}$, Kondo $\mathrm{H}$ et al. Dietary diacylglycerol suppresses high fat and high sucrose diet-induced body fat accumulation in C57BL/6J mice. J Lipid Res 2001; 42: 372-378.

16 Panchal SK, Brown L. Rodent models for metabolic syndrome research. J Biomed Biotechnol 2011; 2011: 351982

17 Parekh PI, Petro AE, Tiller JM, Feinglos MN, Surwit RS. Reversal of diet-induced obesity and diabetes in C57BL/6J mice. Metabolism 1998; 47: 1089-1096.

18 Panchal SK, Poudyal H, lyer A, Nazer R, Alam A, Diwan V et al. High-carbohydrate high-fat diet-induced metabolic syndrome and cardiovascular remodeling in rats. J Cardiovasc Pharmacol 2011; 57: 51-64.

19 Wada T, Kenmochi H, Miyashita $\mathrm{Y}$, Sasaki M, Ojima M, Sasahara M et al. Spironolactone improves glucose and lipid metabolism by ameliorating hepatic steatosis and inflammation and suppressing enhanced gluconeogenesis induced by high-fat and high-fructose diet. Endocrinology 2010; 151: 2040-2049.

20 Ricci MR, Ulman EA. Laboratory animal diets: a critical part of your in vivo research. Anim Lab News 2005; 4: 1-5.

21 Bell RR, Spencer MJ, Sherriff JL. Voluntary exercise and monounsaturated canola oil reduce fat gain in mice fed diets high in fat. J Nutr 1997; 127: 2006-2010.

22 Ikemoto S, Takahashi M, Tsunoda N, Maruyama K, Itakura H, Ezaki O. High-fat diet-induced hyperglycemia and obesity in mice: differential effects of dietary oils. Metabolism 1996; 45: 1539-1546.

23 Gallou-Kabani C, Vige A, Gross MS, Rabes JP, Boileau C, Larue-Achagiotis C et al. C57BL/6J and A/J mice fed a high-fat diet delineate components of metabolic syndrome. Obesity (Silver Spring) 2007; 15: 1996-2005.

24 Buettner R, Parhofer KG, Woenckhaus M, Wrede CE, Kunz-Schughart LA, Scholmerich $J$ et al. Defining high-fat-diet rat models: metabolic and molecular effects of different fat types. J Mol Endocrinol 2006; 36: 485-501.

25 Omar B, Pacini G, Ahren B. Differential development of glucose intolerance and pancreatic islet adaptation in multiple diet induced obesity models. Nutrients 2012; 4: 1367-1381.

26 Warden $\mathrm{CH}$, Fisler JS. Comparisons of diets used in animal models of high-fat feeding. Cell Metab 2008; 7: 277.

27 Torre-Villalvazo I, Tovar AR, Ramos-Barragan VE, Cerbon-Cervantes MA, Torres N. Soy protein ameliorates metabolic abnormalities in liver and adipose tissue of rats fed a high fat diet. J Nutr 2008; 138: 462-468.

28 Martin B, Ji S, Maudsley S, Mattson MP. "Control" laboratory rodents are metabolically morbid: why it matters. Proc Natl Acad Sci USA 2010; 107: 6127-6133.

29 Morrison CD, Huypens P, Stewart LK, Gettys TW. Implications of crosstalk between leptin and insulin signaling during the development of diet-induced obesity. Biochim Biophys Acta 2009; 1792: 409-416.

30 Korou L-MA, Doulamis IP, Tzanetakou IP, Mikhailidis DP, Perrea DN. The effect of biological age on the metabolic responsiveness of mice fed a high-fat diet. Lab Anim 2013; 47: 241-244.

31 de Castro UGM, dos Santos RAS, Silva M, de Lima W, Campagnole-Santos M, Alzamora A. Age-dependent effect of high-fructose and high-fat diets on lipid metabolism and lipid accumulation in liver and kidney of rats. Lipids Health Dis 2013; 12: 136

32 Tschen S-I, Dhawan S, Gurlo T, Bhushan A. Age-dependent decline in $\beta$-cell proliferation restricts the capacity of $\beta$-cell regeneration in mice. Diabetes 2009; 58: $1312-1320$.

33 Schemmel R, Mickelsen O, Gill JL. Dietary obesity in rats: body weight and body fat accretion in seven strains of rats. J Nutr 1970; 100: 1041-1048.

34 West DB, Boozer CN, Moody DL, Atkinson RL. Dietary obesity in nine inbred mouse strains. Am J Physiol 1992; 262(Pt 2): R1025-R1032.

35 Rossmeisl M, Rim JS, Koza RA, Kozak LP. Variation in type 2 diabetes--related traits in mouse strains susceptible to diet-induced obesity. Diabetes 2003; 52 1958-1966.

36 Andrikopoulos S, Massa CM, Aston-Mourney K, Funkat A, Fam BC, Hull RL et al. Differential effect of inbred mouse strain (C57BL/6, DBA/2, 129T2) on insulin secretory function in response to a high fat diet. J Endocrinol 2005; 187: 45-53.

37 Levin BE, Dunn-Meynell AA, Balkan B, Keesey RE. Selective breeding for dietinduced obesity and resistance in Sprague-Dawley rats. Am J Physiol 1997; 273(Pt 2): R725-R730.

38 Chang S, Graham B, Yakubu F, Lin D, Peters JC, Hill JO. Metabolic differences between obesity-prone and obesity-resistant rats. Am J Physiol 1990; 259(Pt 2): R1103-R1110. 
39 Chia R, Achilli F, Festing MF, Fisher EM. The origins and uses of mouse outbred stocks. Nat Genet 2005; 37: 1181-1186.

40 Burcelin R, Crivelli V, Dacosta A, Roy-Tirelli A, Thorens B. Heterogeneous metabolic adaptation of C57BL/6J mice to high-fat diet. Am J Physiol Endocrinol Metab 2002; 282: E834-E842.

41 Svenson KL, Von Smith R, Magnani PA, Suetin HR, Paigen B, Naggert JK et al. Multiple trait measurements in 43 inbred mouse strains capture the phenotypic diversity characteristic of human populations. J Appl Physiol 2007; 102: 2369-2378.

42 Fearnside JF, Dumas M-E, Rothwell AR, Wilder SP, Cloarec O, Toye A et al. Phylometabonomic patterns of adaptation to high fat diet feeding in inbred mice. PLoS One 2008; 3: e1668.

43 Metlakunta AS, Sahu M, Sahu A. Hypothalamic phosphatidylinositol 3-kinase pathway of leptin signaling is impaired during the development of diet-induced obesity in FVB/N mice. Endocrinology 2008; 149: 1121-1128.

44 Boudina S, Sena S, Sloan C, Tebbi A, Han YH, O'Neill BT et al. Early mitochondrial adaptations in skeletal muscle to diet-induced obesity are strain dependent and determine oxidative stress and energy expenditure but not insulin sensitivity. Endocrinology 2012; 153: 2677-2688.

45 Winzell MS, Ahren B. The high-fat diet-fed mouse: a model for studying mechanisms and treatment of impaired glucose tolerance and type 2 diabetes. Diabetes 2004; 53(Suppl 3): S215-S219.

46 Sone H, Kagawa Y. Pancreatic beta cell senescence contributes to the pathogenesis of type 2 diabetes in high-fat diet-induced diabetic mice. Diabetologia 2005; 48: 58-67.

47 Reimer MK, Ahren B. Altered beta-cell distribution of pdx-1 and GLUT-2 after a short-term challenge with a high-fat diet in C57BL/6J mice. Diabetes 2002; 51(Suppl 1): S138-S143.

$48 \mathrm{Wu}$ L, Vikramadithyan R, Yu S, Pau C, Hu Y, Goldberg IJ et al. Addition of dietary fat to cholesterol in the diets of LDL receptor knockout mice: effects on plasma insulin, lipoproteins, and atherosclerosis. J Lipid Res 2006; 47: 2215-2222.

49 Ayala JE, Samuel VT, Morton GJ, Obici S, Croniger CM, Shulman Gl et al. Standard operating procedures for describing and performing metabolic tests of glucose homeostasis in mice. Dis Model Mech 2010; 3: 525-534.

50 Brown ET, Umino Y, Loi T, Solessio E, Barlow R. Anesthesia can cause sustained hyperglycemia in C57/BL6J mice. Vis Neurosci 2005; 22: 615-618.

51 Klempt M, Rathkolb B, Fuchs E, de Angelis MH, Wolf E, Aigner B. Genotypespecific environmental impact on the variance of blood values in inbred and F1 hybrid mice. Mamm Genome 2006; 17: 93-102.

52 Ayala JE, Bracy DP, McGuinness OP, Wasserman DH. Considerations in the design of hyperinsulinemic-euglycemic clamps in the conscious mouse. Diabetes 2006; 55: 390-397.

53 Heijboer AC, Donga E, Voshol PJ, Dang ZC, Havekes LM, Romijn JA et al. Sixteen hours of fasting differentially affects hepatic and muscle insulin sensitivity in mice. J Lipid Res 2005; 46: 582-588.

54 Kohsaka A, Bass J. A sense of time: how molecular clocks organize metabolism. Trends Endocrinol Metab 2007; 18: 4-11.

55 Lenzen S. The mechanisms of alloxan- and streptozotocin-induced diabetes. Diabetologia 2008; 51: 216-226.

56 Reed MJ, Meszaros K, Entes LJ, Claypool MD, Pinkett JG, Gadbois TM et al. A new rat model of type 2 diabetes: the fat-fed, streptozotocin-treated rat. Metabolism 2000; 49: 1390-1394.

57 Srinivasan K, Viswanad B, Asrat L, Kaul CL, Ramarao P. Combination of high-fat diet-fed and low-dose streptozotocin-treated rat: a model for type 2 diabetes and pharmacological screening. Pharmacol Res 2005; 52: 313-320.

58 Eiki J-I, Nagata Y, Futamura M, Sasaki-Yamamoto K, lino T, Nishimura T et al. Pharmacokinetic and pharmacodynamic properties of the glucokinase activator MK-0941 in rodent models of type 2 diabetes and healthy dogs. Mol Pharmacol 2011; 80: 1156-1165.

59 Cao S, Li B, Yi X, Chang B, Zhu B, Lian Z et al. Effects of exercise on AMPK signaling and downstream components to PI3K in rat with type 2 diabetes. PLOS One 2012; 7: e51709.

60 Ding Y, Zhang Z, Dai X, Jiang Y, Bao L, Li Y et al. Grape seed proanthocyanidins ameliorate pancreatic beta-cell dysfunction and death in low-dose streptozotocin- and high-carbohydrate/high-fat diet-induced diabetic rats partially by regulating endoplasmic reticulum stress. Nutr Metab 2013; 10: 51.

$61 \mathrm{Li} \mathrm{Y,} \mathrm{Chen} \mathrm{M,} \mathrm{Xuan} \mathrm{H,} \mathrm{Hu} \mathrm{F.} \mathrm{Effects} \mathrm{of} \mathrm{encapsulated} \mathrm{propolis} \mathrm{on} \mathrm{blood} \mathrm{glycemic}$ control, lipid metabolism, and insulin resistance in type 2 diabetes mellitus rats. Evid Based Complement Alternat Med 2012; 2012: 981896.

62 Zhang M, Lv XY, Li J, Xu ZG, Chen L. The characterization of high-fat diet and multiple low-dose streptozotocin induced type 2 diabetes rat model. Exp Diabetes Res 2008; 2008: 704045.

63 Szkudelski T, Kandulska K, Okulicz M. Alloxan in vivo does not only exert deleterious effects on pancreatic B cells. Physiol Res 1998; 47: 343-346.
64 Thliveris JA, Paz GF, Rempel E, Faiman C. An extra-pancreatic direct effect of streptozotocin on the hypothalamo-hypophyseal-testicular axis in the rat. Anat Anz 1984; 157: 213-219.

65 Kume E, Aruga C, Ishizuka Y, Takahashi K, Miwa S, Itoh M et al. Gene expression profiling in streptozotocin treated mouse liver using DNA microarray. Exp Toxicol Pathol 2005; 56: 235-244.

66 Lee HC, Bonner-Weir S, Weri GC, Leahy JL. Compensatory adaption to partial pancreatectomy in the rat. Endocrinology 1989; 124: 1571-1575.

67 Peshavaria M, Larmie BL, Lausier J, Satish B, Habibovic A, Roskens V et al. Regulation of pancreatic $\beta$-cell regeneration in the normoglycemic $60 \%$ partial-pancreatectomy mouse. Diabetes 2006; 55: 3289-3298.

68 Plachot C, Movassat J, Portha B. Impaired beta-cell regeneration after partial pancreatectomy in the adult Goto-Kakizaki rat, a spontaneous model of type II diabetes. Histochem Cell Biol 2001; 116: 131-139.

69 Menge BA, Tannapfel A, Belyaev O, Drescher R, Muller C, Uhl W et al. Partial pancreatectomy in adult humans does not provoke beta-cell regeneration. Diabetes 2008; 57: 142-149.

70 Chandrasekera PC, Pippin JJ. Of rodents and men: species-specific glucose regulation and type 2 diabetes research. Altex 2014; 31: 157-176.

71 Masiello P. Animal models of type 2 diabetes with reduced pancreatic $\beta$-cell mass. Int J Biochem Cell Biol 2006; 38: 873-893.

72 Von Diemen V, Trindade EN, Trindade MRM. Experimental model to induce obesity in rats. Acta Cir Bras 2006; 21: 425-429.

73 Axen KV, Li X, Fung K, Sclafani A. The VMH-dietary obese rat: a new model of non-insulin-dependent diabetes mellitus. Am J Physiol 1994; 266(Pt 2): R921-R928.

74 King AJF. The use of animal models in diabetes research. Br J Pharmacol 2012; 166: 877-894.

75 Nicolson TJ, Bellomo EA, Wijesekara N, Loder MK, Baldwin JM, Gyulkhandanyan AV et al. Insulin storage and glucose homeostasis in mice null for the granule zinc transporter ZnT8 and studies of the type 2 diabetes-associated variants. Diabetes 2009; 58: 2070-2083.

76 Lemaire K, Ravier MA, Schraenen A, Creemers JW, Van de Plas R, Granvik M et al. Insulin crystallization depends on zinc transporter ZnT8 expression, but is not required for normal glucose homeostasis in mice. Proc Natl Acad Sci USA 2009; 106: 14872-14877.

77 Pound LD, Sarkar SA, Benninger RK, Wang Y, Suwanichkul A, Shadoan MK et al. Deletion of the mouse Slc30a8 gene encoding zinc transporter-8 results in impaired insulin secretion. Biochem J 2009; 421: 371-376.

78 Pound LD, Sarkar SA, Ustione A, Dadi PK, Shadoan MK, Lee CE et al. The physiological effects of deleting the mouse SLC30A8 gene encoding zinc transporter- 8 are influenced by gender and genetic background. PLoS One 2012; 7: e40972.

79 Hardy AB, Wijesekara N, Genkin I, Prentice KJ, Bhattacharjee A, Kong D et al. Effects of high-fat diet feeding on Znt8-null mice: differences between beta-cell and global knockout of Znt8. Am J Physiol Endocrinol Metab 2012; 302: E1084-E1096.

80 Flannick J, Thorleifsson G, Beer NL, Jacobs SB, Grarup N, Burtt NP et al. Loss-of-function mutations in SLC30A8 protect against type 2 diabetes. Nat Genet 2014; 46: 357-363.

81 Steneberg P, Rubins N, Bartoov-Shifman R, Walker MD, Edlund H. The FFA receptor GPR40 links hyperinsulinemia, hepatic steatosis, and impaired glucose homeostasis in mouse. Cell Metab 2005; 1: 245-258.

82 Lan H, Hoos LM, Liu L, Tetzloff G, Hu W, Abbondanzo SJ et al. Lack of FFAR1/GPR40 does not protect mice from high-fat diet-induced metabolic disease. Diabetes 2008; 57: 2999-3006.

83 Bunner AE, Chandrasekera PC, Barnard ND. Knockout mouse models of insulin signaling: Relevance past and future. World J Diabetes 2014; 5: 146-159.

84 Kulkarni RN, Almind K, Goren HJ, Winnay JN, Ueki K, Okada T et al. Impact of genetic background on development of hyperinsulinemia and diabetes in insulin receptor/insulin receptor substrate-1 double heterozygous mice. Diabetes 2003; 52: 1528-1534.

85 Elchebly M, Payette P, Michaliszyn E, Cromlish W, Collins S, Loy AL et al. Increased insulin sensitivity and obesity resistance in mice lacking the protein tyrosine phosphatase-1b gene. Science 1999; 283: 1544-1548.

86 Li T, Owsley E, Matozel M, Hsu P, Novak CM, Chiang JYL. Transgenic expression of cholesterol 7a-hydroxylase in the liver prevents high-fat diet-induced obesity and insulin resistance in mice. Hepatology 2010; 52: 678-690.

87 Hiltunen $\mathrm{M}$, Khandelwal VK, Yaluri N, Tiilikainen $\mathrm{T}$, Tusa $\mathrm{M}$, Koivisto $\mathrm{H}$ et al. Contribution of genetic and dietary insulin resistance to Alzheimer phenotype in APP/PS1 transgenic mice. J Cell Mol Med 2012; 16: 1206-1222.

88 Chalkiadaki A, Guarente L. High-fat diet triggers inflammation-induced cleavage of SIRT1 in adipose tissue to promote metabolic dysfunction. Cell Metab 2012; 16: $180-188$. 
89 Austin GL, Ogden LG, Hill JO. Trends in carbohydrate, fat, and protein intakes and association with energy intake in normal-weight, overweight, and obese individuals: 1971-2006. Am J Clin Nutr 2011; 93: 836-843.

90 Ford ES, Dietz WH. Trends in energy intake among adults in the United States: findings from NHANES. Am J Clin Nutr 2013; 97: 848-853.

91 National Cancer Institute. Food Sources of Energy among U.S. Ppopulation, 2005-2006. Risk Factor Monitoring and Methods. Cancer Control and Population Sciences: Bethesda, MD, USA, 2010. Available from http://riskfactor.cancer.gov/ diet/foodsources/.

92 Fradin D, Bougneres P. T2DM: why epigenetics? J Nutr Metab 2011; 2011: 647514.

93 Halford JC, Boyland EJ, Blundell JE, Kirkham TC, Harrold JA. Pharmacological management of appetite expression in obesity. Nat Rev Endocrinol 2010; 6 : 255-269.

94 Jensen TL, Kiersgaard MK, Sorensen DB, Mikkelsen LF. Fasting of mice: a review. Lab Anim 2013; 47: 225-240.

95 Agouni A, Owen C, Czopek A, Mody N, Delibegovic M. In vivo differential effects of fasting, re-feeding, insulin and insulin stimulation time course on insulin signaling pathway components in peripheral tissues. Biochem Biophys Res Commun 2010; 401: 104-111.

96 Cannon B, Nedergaard J. Nonshivering thermogenesis and its adequate measurement in metabolic studies. J Exp Biol 2011; 214: 242-253.

97 Gaskill BN, Gordon CJ, Pajor EA, Lucas JR, Davis JK, Garner JP. Heat or insulation: behavioral titration of mouse preference for warmth or access to a nest. PLoS One 2012; 7: e32799.

98 Ledford H. Chilly lab mice skew cancer studies. Nature 2013; doi:10.1038/ nature.2013.14190

99 Lodhi IJ, Semenkovich CF. Why we should put clothes on mice. Cell Metab 2009; 9: 111-112.

100 Kahn SE. Clinical review 135: the importance of beta-cell failure in the development and progression of type 2 diabetes. J Clin Endocrinol Metabolism 2001; 86: 4047-4058.

101 Robinson R, Barathi VA, Chaurasia SS, Wong TY, Kern TS. Update on animal models of diabetic retinopathy: from molecular approaches to mice and higher mammals. Dis Model Mech 2012; 5: 444-456.

102 Dissard R, Klein J, Caubet C, Breuil B, Siwy J, Hoffman J et al. Long term metabolic syndrome induced by a high fat high fructose diet leads to minimal renal injury in C57BL/6 mice. PLoS One 2013; 8: e76703.

103 Sullivan KA, Hayes JM, Wiggin TD, Backus C, Su Oh S, Lentz SI et al. Mouse models of diabetic neuropathy. Neurobiol Dis 2007; 28: 276-285.

104 Obrosova IG, Inytska O, Lyzogubov VV, Pavlov IA, Mashtalir N, Nadler JL et al. High-fat diet induced neuropathy of pre-diabetes and obesity: effects of "healthy" diet and aldose reductase inhibition. Diabetes 2007; 56: 2598-2608.

105 Li S, Zhang H-Y, Hu CC, Lawrence F, Gallagher KE, Surapaneni A et al. Assessment of diet-induced obese rats as an obesity model by comparative functional genomics. Obesity 2008; 16: 811-818.

106 Pecoraro N, Ginsberg AB, Warne JP, Gomez F, la Fleur SE, Dallman MF. Diverse basal and stress-related phenotypes of Sprague Dawley rats from three vendors. Physiol Behav 2006; 89: 598-610.
107 Tsilidis KK, Panagiotou OA, Sena ES, Aretouli E, Evangelou E, Howells DW et al. Evaluation of excess significance bias in animal studies of neurological diseases. PLoS Biol 2013; 11: e1001609.

108 Sena ES, van der Worp HB, Bath PM, Howells DW, Macleod MR. Publication bias in reports of animal stroke studies leads to major overstatement of efficacy. PLoS Biol 2010; 8: e1000344.

109 Wang CC, Adochio RL, Leitner JW, Abeyta IM, Draznin B, Cornier MA. Acute effects of different diet compositions on skeletal muscle insulin signalling in obese individuals during caloric restriction. Metabolism 2013; 62 595-603.

110 Ruiz-Alcaraz AJ, Lipina C, Petrie JR, Murphy MJ, Morris AD, Sutherland C et al. Obesity-induced insulin resistance in human skeletal muscle is characterised by defective activation of p42/p44 MAP kinase. PLoS One 2013; 8: e56928.

111 Hwang $H$, Bowen BP, Lefort N, Flynn CR, De Filippis EA, Roberts C et al. Proteomics analysis of human skeletal muscle reveals novel abnormalities in obesity and type 2 diabetes. Diabetes 2010; 59: 33-42.

112 Volkmar M, Dedeurwaerder S, Cunha DA, Ndlovu MN, Defrance M, Deplus R et al. DNA methylation profiling identifies epigenetic dysregulation in pancreatic islets from type 2 diabetic patients. EMBO J 2012; 31: 1405-1426.

113 Pavkov ME, Hanson RL, Knowler WC, Bennett PH, Krakoff J, Nelson RG. Changing patterns of type 2 diabetes incidence among Pima Indians. Diabetes Care 2007 30: $1758-1763$.

114 Wang H, Wang J, Liu MM, Wang D, Liu YQ, Zhao Y et al. Epidemiology of general obesity, abdominal obesity and related risk factors in urban adults from 33 communities of Northeast China: the CHPSNE study. BMC Public Health 2012; 12: 967.

115 Naja F, Nasreddine L, Itani L, Adra N, Sibai AM, Hwalla N. Association between dietary patterns and the risk of metabolic syndrome among Lebanese adults. Eur J Nutr 2013; 52: 97-105.

116 Odegaard AO, Koh WP, Yuan J-M, Gross MD, Pereira MA. Western-style fast food intake and cardio-metabolic risk in an Eastern country. Circulation 2012; 126: 182-188.

117 Campbell TC, Campbell TM. The China Study: The Most Comprehensive Study of Nutrition Ever Conducted and the Startling Implications for Diet, Weight Loss and Long-Term Health. Wakefield Press: Kent Town, South Australia, Australia, 2007.

118 Ley RE, Turnbaugh PJ, Klein S, Gordon Jl. Microbial ecology: human gut microbes associated with obesity. Nature 2006; 444: 1022-1023.

119 Serino M, Fernandez-Real JM, Fuentes EG, Queipo-Ortuno M, Moreno-Navarrete $J M$, Sanchez A et al. The gut microbiota profile is associated with insulin action in humans. Acta Diabetol 2013; 50: 753-761.

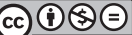

This work is licensed under a Creative Commons AttributionNonCommercial-NoDerivs 4.0 International License. The images or other third party material in this article are included in the article's Creative Commons license, unless indicated otherwise in the credit line; if the material is not included under the Creative Commons license, users will need to obtain permission from the license holder to reproduce the material. To view a copy of this license, visit http:// creativecommons.org/licenses/by-nc-nd/4.0/ 